\title{
Wind Speed Prediction Based on Time series Neural Network Algorithm
}

\author{
Zhaoyang Wang \\ North China Electric Power University ,Baoding 071000, China \\ wang123@163.com
}

Keywords: WindSPeed, TimeSeries, Neural Network, PredietionModels, BP, RBF

\begin{abstract}
This paper choose short-term prediction as the research content of the wind speed,the time series and neural network theory are uesd into to the wind speed prediction model.On the basis of BP model, ARMA-BP model is proposed.Then the ARMA-RBF model was established, makes the prediction error smaller.Simulation experimental results shows that using neural network to establish the sequence of the network, training time significantly shortened, network between predicted values and the real observation values of output doesn't appear too big deviation, the new training sample set fitting is good ,and prediction accuracy improved.
\end{abstract}

\section{Introduction}

China is a big energy country, but also a major energy consuming country. For the use of renewable energy, especially the use of wind energy has become a hot topic of concern.Because wind power has a strong randomness, when the proportion of wind power to the entire grid exceeds the certain value, it will seriously affect the power quality and wind power system operation.Accurate wind speed prediction can effectively reduce or avoid the negative impact of the wind farm on the power system, which is conducive to the timely adjustment of the dispatching plan of the power system dispatching department and improve the competitiveness of the wind power plant in the electricity market.It is hoped that the neural network model and the time series model should be combined to improve the short-term forecasting method and optimize the grid dispatching and resource allocation.

\section{BP model}

The BP (Back Propagation) neural network model was proposed by the team of scientists headed by Rumelhart and McCelland in 1986 and is a multilayer feedforward network trained by error back propagation algorithm. The BP neural network can learn and store a large number of input-output pattern mappings without revealing the mathematical equations describing the mapping relationships beforehand.Its learning rule is to use the steepest descent method, through the communication to constantly adjust the network weights and thresholds, so that the network error square sum and minimum.But BP neural network algorithm learning speed is slow, and easy to fall into the local minimum point, in the design process are often repeated trial and training.Therefore it is difficult to guarantee the convergence and global optimality of the BP neural network algorithm for each training.

\section{ARMA RB Neural Network Model}

\subsection{Establishment of ARMA - BP Neural Network Model}

\subsubsection{Initial data stability processing}

In the time series modeling, the original data to the white noise test firstly. Only the original data is not white noise, modeling is meaningful. And then the data is processed smoothly. There are many common methods of data processing, where the use of differential methods for processing. Using the econometric software EViews, the Dickey - Fuller (ADF) method is used to test the smoothness of the data. 


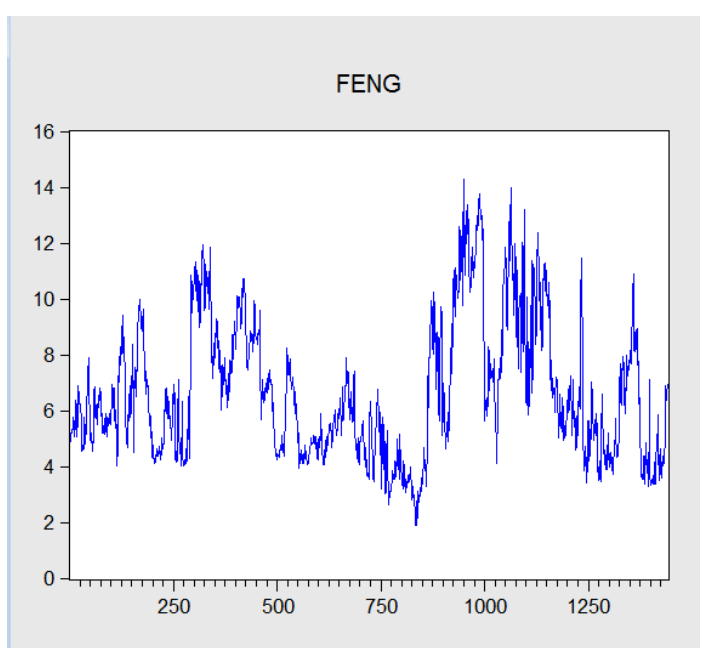

Fig.1 Initial data

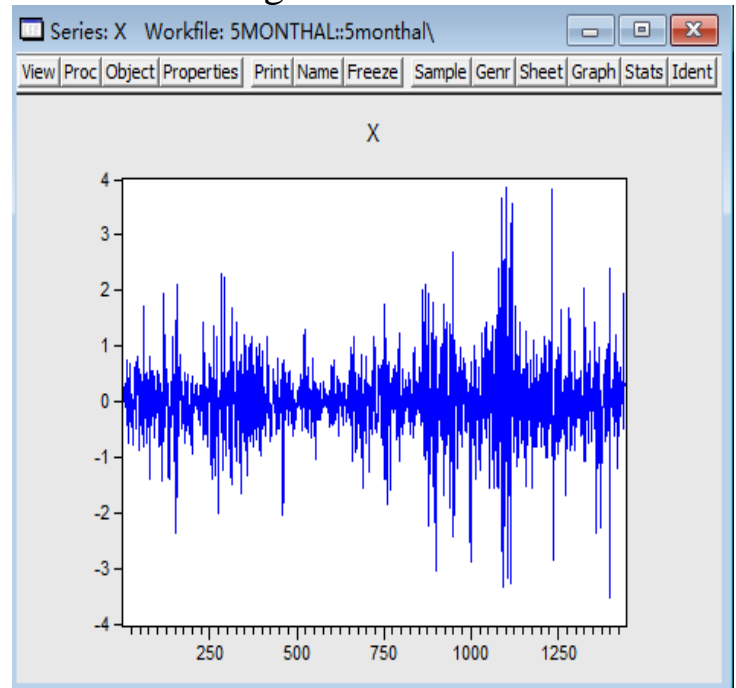

Fig.2 The data after the first-order difference processing

\subsubsection{Model identification}

The recognition of the model is based on the characteristics of the autocorrelation and partial correlation function of the time series, and the type of the model is selected and the model order of the time series is determined. The first step is to calculate the autocorrelation function ACF and the partial correlation function PACF from the time series samples and determine whether the basic requirements of the ARMA (p, q) model are satisfied.

That is: to meet the AR relationship:

$$
\left\{\begin{array}{l}
a_{k j}=\varphi_{j} \quad 1 \leqslant j \leqslant p, \\
a_{k j}=0 \quad j=p+1, p+2, \cdots k ;
\end{array}\right.
$$

to meet the MA relationship:

$$
\rho_{k}= \begin{cases}1 & k=0, \\ \frac{-\theta_{k}+\theta_{k+1} \theta_{1}+\cdots \theta_{q} \theta_{q-k}}{1+\theta_{1}^{2}+\cdots \theta_{q}^{2}} & 1 \leqslant k \leqslant q, \\ 0 & k>q 。\end{cases}
$$

\subsubsection{Parameter estimation of the model}

After the model is identified, it is necessary to determine the order of the model. In this paper, the optimal criterion function is used to determine the order of the model, according to the AIC criterion.

According to the time series method, the difference sequence $\{\mathrm{Zt}\}$ of an original wind speed sequence can be identified as the ARMA (p, q) model, the predicted value Zt (1) is the linear combination.of $\mathrm{p}$ historical value of $\{\mathrm{Zt}\}$ and the nearest $\mathrm{q}$ residual Therefore, the most recent $\mathrm{p}$ 
historical values and the nearest q residual values of the difference sequence $\{\mathrm{Zt}\}$ are selected as the input of the neural network, and the value of $\{\mathrm{Zt}\}$ is output as the target of the network at the next moment.

Here, the ARMA $(4,3)$ model is established based on the sample data.

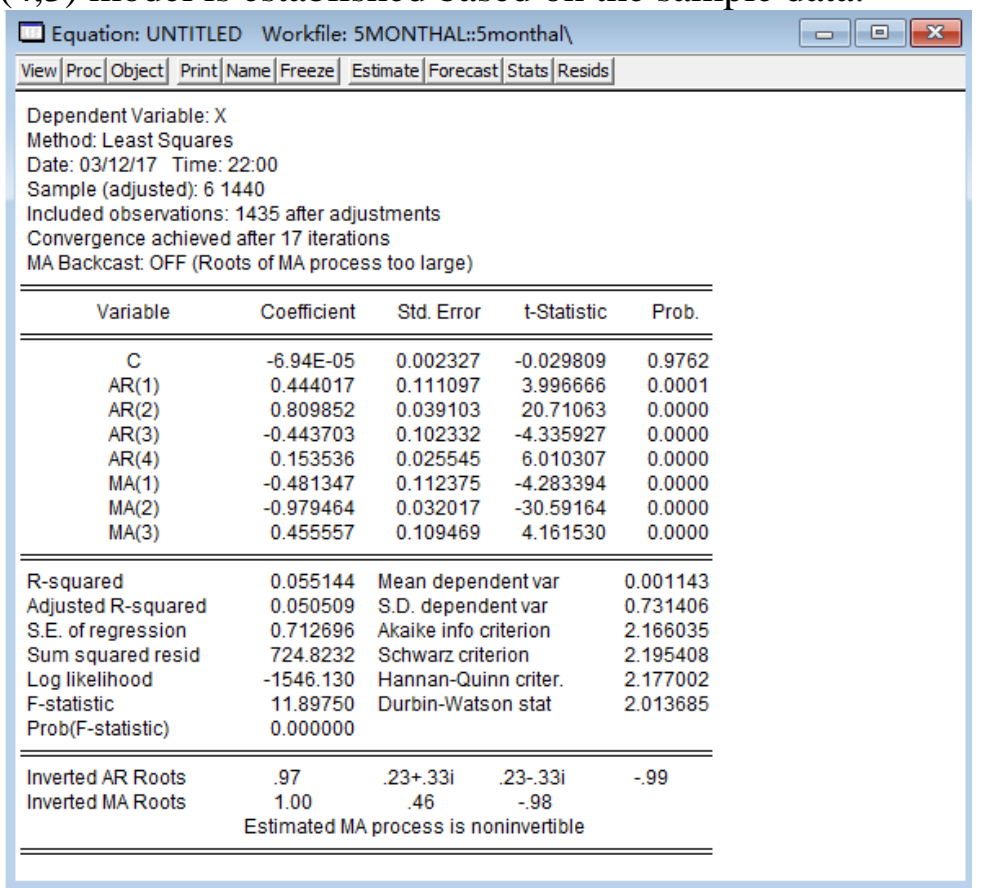

\subsubsection{Model test}

Fig.3 Modeling results

If the $\mathrm{p}$ value is less than 0.5 , then the modeling results through the parameter significance test, in Figure 3, you can see the p value is 0 , so passed the test. Also, do residual white noise test. If the residual is white noise, it means that the data is extracted more fully.

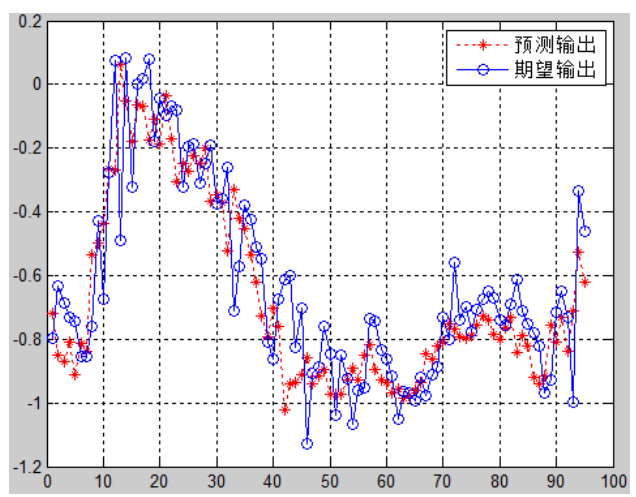

Fig.4 Forecast result

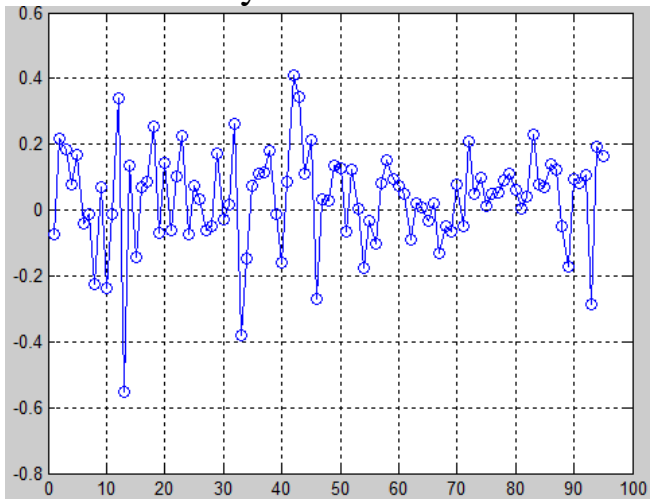

Fig.5 Residual

In addition, the number of iterations of the ARMA-BP model is much less than that of the BP model, and the training time is shorter.

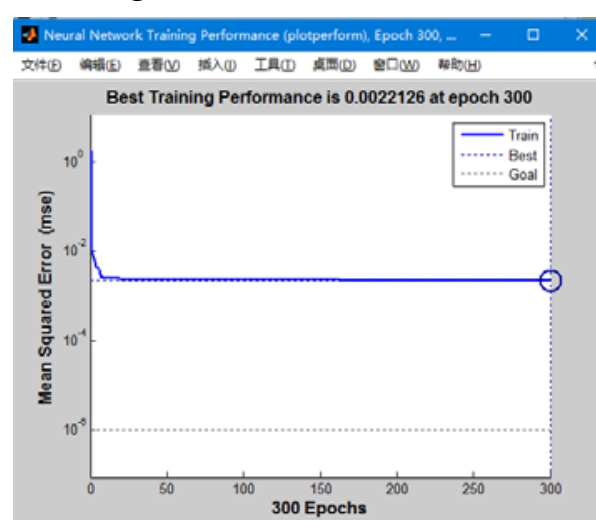

Fig.6 epoch of BP

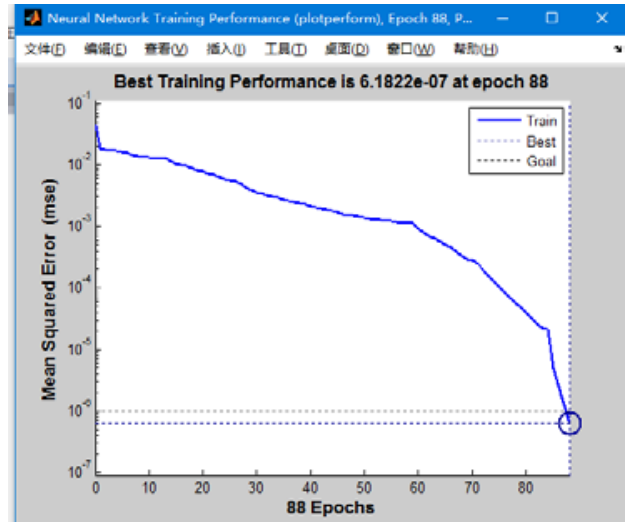

Fig.7 epoch of ARMA-BP 


\section{ARMA RBF Neural Network Model}

As BP neural network algorithm is easy to fall into the minimum point, and the network parameter selection is complicated. Here the RBF neural network is used to optimize the algorithm. Compared with BP network, RBF neural network learning rules are simple, only need to modify the hidden layer and the output layer between the weight, eliminating the need to calculate the error between the layers of the process, the following is the ARMA-RBF model prediction result.As can be seen from the results, the prediction error is smaller, the number of iterations is less and the precision is higher.

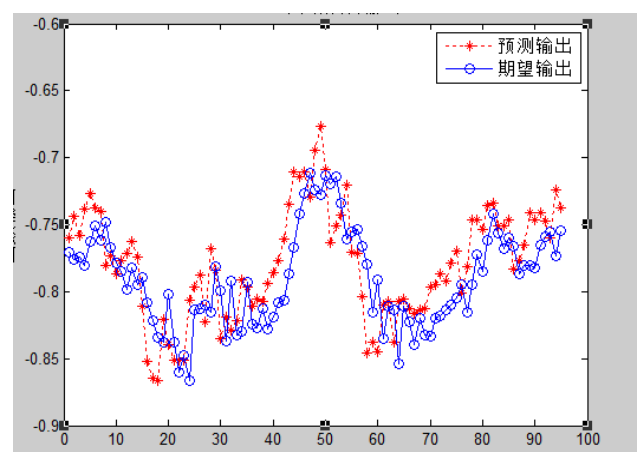

Fig.8 forecast result

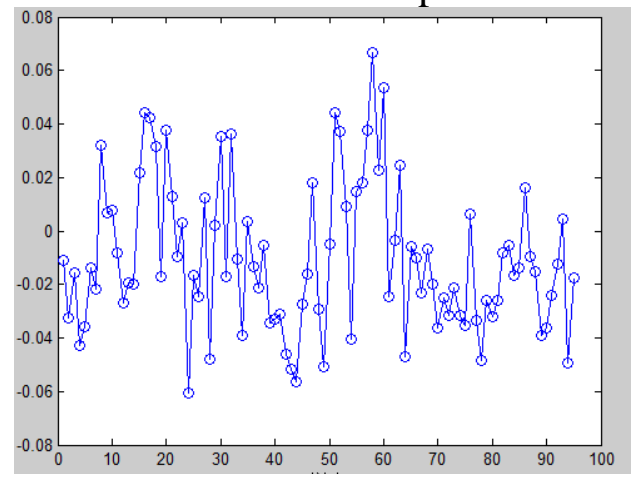

Fig.9 Residual

\section{Conclusions}

Accurate wind speed prediction data is an indispensable reference data in the prediction of wind power generation. A more accurate prediction of wind speed can effectively reduce or avoid the adverse effects of wind farms on the power system. The time series combined with the neural network algorithm greatly reduces the training time and the prediction error is also reduced. Therefore, the time series neural network method is effective to improve the accuracy of wind speed prediction, and has certain practical value. But the wind speed forecast by the temperature, humidity and other factors greater impact, more accurate model to be introduced into the network input factors to achieve more accurate prediction.

\section{References}

[1]. Wind Force 120, the EuropeanWind EnergyAssociation (EWEA),

[2]. October2002,http: //www. ewea. org/doc/WindForce12.pd.f

[3]. DongAnzheng, Zhao Guofan.TheApplication of artificialneural networks in shor-t tmi ewind speed prediction[J]. EngineeringMechanics, 2003,20(5):10-13.

[4]. XiaoYongshan,WangWeiqing,Huo Xiaoping. The forecast research of wind electric field wind speed time series based on neural network

[5]. Energy-saving technologies, 2007, 25(2): 106-108.

[6]. Dong Cong, Zhengneng, Xia Renwe.i The research development and certain questions onMult-i layered predecessor network[ J]. Power system and clean energy, 1995, 35(2): 186-195.

[7]. Liang Lanzhen, ShaoFan.W ind speed short-term forecast forwind farms based on ARMA model[ J]. Power System and Clean Energy,2008, 24(108): 52-55. 